\title{
Perfiles motivacionales de usuarios de servicios deportivos públicos y privados*
}

\section{Motivational Profiles of Users of Private and Public Sport Services}

Recibido: mayo 20 de 2011 |Revisado: febrero 10 de 2012 | Aceptado: agosto 15 de 2012

\author{
Alberto Nuviala Nuviala ** \\ Universidad Pablo de Olavide, Sevilla, España \\ MANUEL GÓMEZ-LÓPEZ *** \\ Universidad de Murcia, España \\ Alberto GraO-CRUCES **** \\ Universidad Pablo de Olavide, Sevilla, España \\ ANTONIO GRANERO-GALLEGOS ***** \\ Universidad de Murcia, España \\ ROMÁN NUVIALA NUVIALA ******* \\ Universidad Pablo de Olavide, Sevilla, España
}

doi:10.11144/Javeriana.UPSY12-2.pmsd

Para citar este artículo: Nuviala, A., Gómez-López, M., Grao-Cruces, A., Granero-Gallegos, A. \& Nuviala, R. (2013). Perfiles motivacionales de usuarios de servicios deportivos públicos y privados. Universitas Psychologica, 12(2), 421-431.

* Esta investigación forma parte de la tesis doctoral de Román Nuviala Nuviala, realizada sobre servicios deportivos y la valoración que hacen sus usuarios.

* Profesor Titular de la Universidad Pablo de Olavide.E-mail: anuvnvuv@upo.es

*** Profesor Contratado. Doctor Universidad de Murcia. E-mail: mgomezlop@um.es

****** Becario de postgrado. Programa de Formación de Profesorado Universitario. Ministerio de Educación. Gobierno de España. E-mail: agracru@ upo.es

******* Profesor Contratado. Doctor Universidad de Murcia.E-mail: agranero@um.es

${ }^{* * * * * * *}$ Doctor del grupo de investigación SEJ-046 de la Universidad Pablo de Olavide. E-mail: romanescarbo@hotmail.com

\section{RESUMEN}

Las altas tasas de sedentarismo y abandono deportivo y la escasez de estudios sobre motivos de práctica en el ámbito de la actividad física no competitiva han generado la necesidad de llevar a cabo investigaciones que establezcan perfiles motivacionales de usuarios de servicios deportivos. La finalidad de esta investigación fue diferenciar los perfiles motivacionales con respecto a la práctica físico-deportiva y analizar su relación con variables sociodemográficas (edad y sexo). La muestra del estudio estuvo compuesta por un total de 2707 usuarios de servicios deportivos, públicos y privados, de Andalucía (España), que cumplimentaron la escala de Motivos de Actividad Física-Revisada (Motives for Physical Activity Measure-Revised [MPAM-R]). Se realizó un análisis por conglomerados y, seguidamente, la prueba ji-cuadrado. Los resultados obtenidos en este análisis establecieron la existencia de tres perfiles motivacionales: uno altamente motivado, especialmente hacia la salud, con sujetos principalmente varones jóvenes de organizaciones públicas; un segundo grupo con motivación por debajo de la media en todas sus dimensiones, e inducido, especialmente por razones de salud, disfrute y apariencia, con un perfil de usuario privado, mujer, de edad media o avanzada; un tercer grupo en el que primaron los motivos sociales y de disfrute, conformado mayoritariamente por usuarios de organizaciones privadas y de sexo masculino.

Palabras clave autores

Motivos, deporte, actividad física, salud, fitness.

Palabras clave descriptores

Investigación cuantitativa, psicología del deporte, Motivación.

\section{A B S T R ACT}

High rates of inactivity and sport neglect and the lack of studies on the basis of practicing in the field of non-competitive physical activity has generated the need to carry out researches to establish motivational profiles in the users of sport services. The purpose of this research was to differentiate motivational profiles respect to physical and sport practice and analyze its relationship with sociodemographic variables (age and sex). The study sample comprised a total of 2707 users of sports services, public and private, of Andalusia (Spain), who completed scale Motives for Physical Activity Measure-Revised (MPAM-R). A cluster analysis was performed an then 
ji-square test. The results of this analysis established the existence of three motivational profiles: a highly motivated, especially on health, mainly young male subjects of public organizations, a second group with motivation below average in all its dimensions, and induced especially for health, taste and appearance reasons, with a private user profile, female, middle or advanced age, a third group in which social and enjoyment motives prevailed, composed mainly by users of private sport services and male.

Key words authors

Motives, Sport, Physical Activity, Health, Fitness.

Key words plus

Quantitative Research, Sport Psychology, Motivation.

\section{Introducción}

En la actualidad, los problemas de salud derivados de la falta de actividad física (AF) ocupan un lugar preferente, tanto en países desarrollados como en vías de desarrollo (Guthold, Ono, Strong, Chatterji \& Morabia, 2008; Organisation for Economic Cooperation and Development [OECD], 2010). En los últimos años se han puesto en práctica en estos países diferentes políticas de promoción de la AF desde diversos ámbitos (Aranceta et al., 2007; Lobstein $\&$ Millstone, 2007). Sin embargo, estas medidas no parecen lo suficientemente eficaces para contrarrestar los problemas para iniciarse y adherirse a la práctica físicodeportiva regular, reflejándose en unos altos índices de sedentarismo (García, 2010; OECD, 2010; Otero, 2009). Existe, por tanto, la necesidad de conocer el comportamiento y los motivos de práctica de la población para mejorar la eficacia de estas iniciativas (Deci \& Ryan, 2000; Rhodes \& De Brujin, 2010).

La relación entre aspectos afectivos y motivacionales con la práctica de AF está bien documentada (Conroy, Hyde, Doerksen \& Ribeiro, 2010; Rhodes, Fiala \& Conner, 2009); se conoce que la orientación motivacional no solo guarda relación con el inicio de la práctica de $\mathrm{AF}$, sino que repercute en mayor medida sobre la adherencia y abandono de esta (Deci \& Ryan, 2000), de ahí que se haya despertado recientemente un interés por encontrar perfiles motivacionales a este respecto (Moreno, González-Cutre \& Cervelló, 2008; Moreno, Martínez-Galindo, González-Cutre \& Marcos, 2009; Sicilia, Águila, Muyor, Orta \& Mo- reno, 2009). Es conocida la asociación positiva de la adherencia con motivos de práctica de disfrute, competencia y social, y negativa con motivos de práctica relacionados con la apariencia o la salud (Frederick-Recascino \& Shuster-Schmidt, 2003; Ryan, Frederick, Lepes, Rubio \& Sheldon, 1997), dimensiones de la escala Motives for Physical Activity Measure-Revised (MPAM-R) de Ryan et al. (1997), la cual se ha validado recientemente en España (Moreno, Cervelló \& Martínez-Camacho, 2007).

Moreno et al. (2007) enmarcan la escala MPAM- $R$ dentro de un enfoque que concibe la motivación como resultado de diferentes variables de índole individual, social y ambiental, que determina, en el caso de la práctica físico-deportiva, la actividad escogida, la intensidad y persistencia en la práctica de esa actividad y el rendimiento en su desempeño. Esta escala está compuesta por cinco factores sobre motivos de Disfrute (p. ej., "Realizo actividad física porque es divertido”), Apariencia (p. ej., "Realizo actividad física porque quiero mantener mi peso para mantener una buena imagen”), Social (p. ej., "Realizo actividad física porque quiero conocer a nuevas personas"), Fitness/Salud (p. ej., "Realizo actividad física porque quiero mejorar mi aptitud cardiovascular") y Competencia (p. ej., "Realizo actividad física porque me gustan los desafíos), de los que Disfrute y Competencia reflejan niveles de motivación intrínseca y los tres restantes han sido utilizados para reflejar una orientación motivacional extrínseca. Estos autores respaldan la escala MPAM-R por ser el instrumento de medida de los motivos de práctica físico-deportiva más completo en sujetos participantes en AF no competitiva.

Una revisión de la literatura muestra que son escasos los estudios sobre motivos de práctica en el ámbito concreto de la AF no competitiva (GraneroGallegos, Gómez-López, Abraldes \& RodríguezSuárez, 2011; Moreno et al., 2007), pese a ser este tipo de AF la más extendida en la población, el 74 \% de la española y en constante incremento (García, 2010). Además, gran parte de estos estudios se han centrado en una franja reducida de edad (Castillo, Balaguer \& Duda, 2000; Kilpatrick, Hebert \& Bartholomew, 2005; Olmand, Daley \& Richard, 2004; Yan \& McCullagh, 2004), no resultando sencillo 
encontrar estudios recientes, publicados en revistas con ciertos indicadores de calidad, que atiendan a muestras considerables con grandes rangos de edad, máxime si se habla exclusivamente de usuarios de servicios deportivos. No obstante, sí ha sido documentado el dinamismo temporal de estos motivos (Beaton \& Funk, 2008) y su relación con variables sociodemográficas, tales como la edad y el sexo (Moreno et al., 2007; Ruiz, García \& Díaz, 2007), pudiendo variar en función del lugar geográfico (Koo \& Hardin, 2008; Wann, Grieve, Zapalac \& Pease, 2008; Won \& Kitamura, 2007).

La motivación de la juventud hacia la práctica de AF tiene un enfoque vitalista, centrado en el disfrute principalmente (Castillo et al., 2000; García, 2006; Hellín, Moreno \& Rodríguez, 2004; Nuviala, Ruiz, García \& Díaz, 2006; Otero, 2009; Ruiz et al., 2007), sin olvidar el papel de los aspectos sociales (García, 2006), de apariencia (Kilpatrick et al., 2005; Olmand et al., 2004) y en menor medida de competencia (García, 2006; Hellín et al., 2004; Moreno et al., 2007; Otero, 2009), a los que se debe incluir la salud como factor de motivación relevante en estas edades (Castillo et al., 2000; Fraile \& De Diego, 2006; Nuviala et al., 2006; Ruiz et al., 2007). Motivos que varían en importancia con el paso del tiempo en las personas, tendiendo a ocupar un lugar más predominante la salud (García, 2006; Hellín et al., 2004; Moreno et al., 2007; Otero, 2009; Ruiz et al., 2007; Sit, Kerr \& Wong, 2008), aunque ese cambio no es tan claro en varones (Otero, 2009).

De hecho, se conoce que el sexo influye en los motivos de práctica de AF; en los varones prima el disfrute $y$, secundariamente, factores sociales, mientras que en las mujeres existe una mayor preocupación por la apariencia y la salud (García, 2006; Hellín et al., 2004; Otero, 2009), aunque algunos estudios han encontrado que estas diferencias están tendiendo a desaparecer (Otero, 2009), o han sido cuestionadas como en el caso de Moreno et al. (2007) quienes encuentran una puntuación ligeramente superior en los motivos de salud que en los de disfrute en ambos sexos; por su parte, Sit et al. (2008) han puesto de manifiesto que el placer, junto con la salud, es el principal motivo de práctica del sexo femenino y Ruiz et al. (2007), a pesar de coincidir en el hecho de que los motivos de disfrute primen en el sexo masculino y los de salud en el femenino, que hallaron que ambos fueron los principales motivos de práctica para los dos sexos.

La variable nivel de estudios también ha sido relacionada con la práctica de $\mathrm{AF}$, resultando una mayor práctica conforme el nivel de estudios es mayor (Breuer, Hallmann, Wicker \& Feiler, 2010; García, 2006; Otero, 2009). Sin embargo, no se tiene constancia de ningún trabajo que haya atendido a la relación del nivel de estudios con los motivos de práctica físico deportiva.

Pese a haberse documentado que existen diferencias entre los usuarios de servicios deportivos públicos y privados en función de algunas de las variables antes expuestas -los más jóvenes y con mayor nivel de estudios optan en mayor grado por las privadas con respecto a los usuarios de mayor edad y menor nivel cultural- (Otero, 2009), se desconoce el perfil de motivación hacia la práctica que pueda tener un usuario de una organización pública en contraste al de una organización privada. Información fundamental para la gestión deportiva, dado que el éxito de esta radica sustancialmente en adaptarse a la diversidad de intereses y motivaciones de sus usuarios (Funk, Filo, Beaton \& Pritchard, 2009), lo que no está ocurriendo en diversos puntos de la geografía española (Hellín et al., 2004). Especial relevancia tendría esa información en la comunidad andaluza donde, en contra de lo que sucede en el conjunto del Estado (García, 2010), la mayor parte de su población activa practica AF de manera organizada (Otero, 2009). Dicho esto, cabe destacar que por organización pública, o servicio deportivo público, el presente artículo hace referencia a aquellas entidades dependientes del Estado y destinadas a prestar un servicio a la ciudadanía. Mientras que por organización privada se refiere a las que no forman parte de la administración estatal, autonómica o local, y cuya finalidad habitual es obtener beneficios económicos con el servicio deportivo que ofertan.

Moreno et al. (2007) sostienen que se precisan nuevas investigaciones sobre la temática que ayuden a diferenciar los contextos de práctica. Por ello y por la aplicación práctica que tienen los resultados de 
esta línea de investigación, tanto para el márquetin y gestión de los servicios deportivos, como para la promoción de la AF, se establecieron los siguientes objetivos: diferenciar los perfiles de motivación hacia la práctica en la población usuaria de servicios deportivos en Andalucía y relacionar los perfiles motivacionales con el tipo de organización, el nivel educativo, el sexo y la edad de los usuarios.

\section{Método}

\section{Diseño del estudio}

Se empleó una metodología de estudio descriptiva, de corte transversal, en el que se han establecido perfiles motivacionales de usuarios de servicios deportivos públicos y privados.

\section{Participantes}

La muestra estuvo compuesta por 2.707 usuarios de distintos servicios deportivos de Andalucía (España), de los cuales el $66.1 \%$ eran varones y el $33.9 \%$ mujeres, con una edad media de $25.29 \pm 12.83$. Los usuarios fueron seleccionados al azar en 78 organizaciones, 24 de ellas eran de ámbito público. Esta decisión permitió afirmar que se ha trabajado con un margen de error de $\pm 1.92 \%$, para un nivel de confianza del $95 \%$.

\section{Instrumentos}

Se utilizó la escala MPAM-R (Ryan et al., 1997), traducida, adaptada y validada al español por Moreno et al. (2007). Este instrumento mide los motivos de práctica hacia la actividad física a través de 28 ítems, agrupados en cinco factores: Disfrute, Apariencia, Social, Fitness/Salud y Competencia. De las cinco subescalas, Disfrute y Competencia han sido utilizadas para reflejar una orientación motivacional intrínseca, mientras que las otras tres subescalas (Social, Fitness/Salud y Apariencia) reflejan varios niveles de motivación extrínseca. Los participantes han respondido a la pregunta "Realizo actividad física..." a través de los ítems que conforman la escala tipo Likert de cinco puntos donde 1 corresponde a nada verdadero para mí y 5 a totalmente verdadero para mí. Los valores de alfa de Cronbach obtenidos fueron: 0.906 para la escala, 0.787 para el factor Disfrute, 0.856 para el factor Apariencia, 0.753 para el factor Social, 0.779 para el factor Fitness/Salud y 0.763 para el factor Competencia. Al cuestionario se le añadieron preguntas sociodemográficas referentes al sexo, edad y estudios.

Esta escala ha sido utilizada y validada por otros autores en distintos contextos (Frederick-Recascino \& Schuster-Smith, 2003), obteniendo en todos ellos una adecuada fiabilidad y validez. A modo de ejemplo, citar que Ryan et al. (1997) obtuvieron los siguientes valores de alfa de Cronbach: Disfrute, $\alpha=0.92$, Apariencia, $\alpha=0.91$, Social, $\alpha=0.83$, Fitness/Salud, $\alpha=0.78 \alpha=0.88$. Del mismo modo, en España, Moreno et al. (2007) obtuvieron los siguientes coeficientes de consistencia interna: Disfrute, $\alpha=0.84$; Apariencia, $\alpha=0.87$; Social, $\alpha=0.81$; Fitness/Salud, $\alpha=0.8$; Competencia, $\alpha=0.85, \mathrm{y}$ Granero-Gallegos et al. (2011) $\alpha=0.84, \alpha=0.89$, $\alpha=0.77, \alpha=0.8$ y $\alpha=0.88$, respectivamente.

\section{Procedimiento}

El trabajo de campo se realizó mediante un cuestionario autoadministrado con presencia del encuestador. Se solicitó a los participantes que lo cumplimentaran y que consultaran cualquier duda que tuvieran con los ítems. El tiempo invertido en la realización fue de unos 10 minutos. Antes de proceder a la recogida de datos se pidió permiso a los responsables de las diferentes organizaciones que participaron en el estudio. De la misma forma, todos los deportistas aceptaron participar voluntariamente en este.

\section{Análisis estadísticos}

Se realizó un análisis de conglomerados (clúster), técnica multivariante que busca agrupar elementos (o variables) tratando de lograr la máxima homogeneidad en cada grupo y las mayores diferencias entre ellos. Se llevó a cabo en primer lugar un análisis clúster jerárquico exploratorio, con el método de Ward, y con base en la lectura del dendograma 
TABLA 1

Distancias entre los centros de los clusters después de aplicar el método k-medias

\begin{tabular}{cccc}
\hline Clúster & 1 & 2 & 3 \\
\hline 1 & & 2.281 & 1.93 \\
2 & 2.281 & & 1.558 \\
3 & 1.93 & 1.558 & \\
\hline
\end{tabular}

Fuente: elaboración propia.

y a los resultados lógicos que se obtuvieron, se seleccionó una solución. Seguidamente, se realizó un segundo análisis clúster a través del método de $k$-medias, utilizando las medias de los clústeres obtenidos como el centro inicial de cada clúster. Finalmente, para conocer la composición social de los grupos se aplicó la prueba de ji-cuadrado $\left(\chi^{2}\right)$ con el objeto de diferenciar entre los diferentes grupos.

Los motivos de práctica, a través de las valoraciones otorgadas a cada una de las dimensiones que se contemplan en el presente estudio (Disfrute, Apariencia, Social, Fitness/Salud y Competencia), fueron las variables utilizadas para establecer los clústeres. En el $\chi^{2}$ se utilizaron como variables dependientes los clústeres resultantes y como variables independientes el tipo de organización (pública o privada), el sexo (varón o mujer), la edad ( $\leq 15,16-24,25-45$ y $>45$ años) y el nivel de estudios (primarios, secundarios o superiores). Para todos los análisis se utilizó el paquete informático SPSS 17.0 para Windows, y se estableció un nivel de significación del $95 \%$.

\section{Resultados}

Los estadísticos descriptivos generados por el total de la muestra revelaron que los usuarios de los servicios deportivos estudiados estaban motivados principalmente hacia la práctica físico-deportiva por razones relacionadas con el factor Disfrute $(M$ = 3.93). Los motivos relacionados con la dimensión Fitness/Salud ( $M=3.84)$ fueron, exceptuando a los de disfrute, más apoyados que los del resto de factores. Este grupo de motivos fueron seguidos por los del factor Social $(M=3.70)$. Aunque en menor medida, los motivos agrupados en los factores Competencia $(M=3.51)$ y Apariencia $(M=3.31)$ fueron destacados como motivos para la práctica de AF no competitiva con puntuaciones superiores a tres en una escala sobre cinco.

Para examinar los perfiles motivacionales mediante la homogenización de los motivos de práctica físico-deportiva se realizó el análisis de conglomerados. Al aplicar el método jerárquico de Ward, con base en la lectura del dendograma y en los resultados lógicos que se obtuvieron, se comprobó la existencia de tres clústeres que presentaban diferentes combinaciones de los factores motivacionales hacia la práctica físico-deportiva. Con la posterior aplicación del método $k$-medias se obtuvieron tres clústeres que presentaron una correcta variabilidad y que estuvieron bien diferenciados entre sí, como ilustran las Tablas 1 y 2.

Como se puede apreciar en la Tabla 2, es el factor Apariencia, con una prueba F $(2,2323)=1289.187$, seguido del factor Social, F $(2,2323)=1108.382$, los que contribuyen en mayor medida a establecer diferencias entre grupos. Es el factor Fitness/Salud, $F(2,2323)=653.141$, el que ha contribuido a la diferenciación en menor medida.

El grupo 1, con un $42.7 \%$ de la muestra, se caracterizó por haber sido el conglomerado con mayor

TABLA 2

Variabilidad de las pruebas F de las cinco dimensiones en los tres clusters obtenidos después de aplicar el método k-medias

\begin{tabular}{lcccccc}
\hline & \multicolumn{2}{c}{ Conglomerado } & \multicolumn{2}{c}{ Error } & \multirow{2}{*}{ F } & Sig. \\
\cline { 2 - 5 } & Media cuadrática & gl & Media cuadrática & gl & & $<0.001$ \\
Disfrute & 176.012 & 2 & 0.207 & 2323 & 848.885 & $<0.001$ \\
Apariencia & 478.955 & 2 & 0.372 & 2323 & 1289.187 & $<0.001$ \\
Social & 357.088 & 2 & 0.322 & 2323 & 1108.382 & $<0.001$ \\
Fitness/Salud & 209.689 & 2 & 0.321 & 2323 & 653.141 & $<0.001$ \\
Competencia & 268.605 & 2 & 0.344 & 2323 & 780.964 & $<0.001$ \\
\hline
\end{tabular}

Fuente: elaboración propia. 
TABLA 3

Centros de los conglomerados y media del factor

\begin{tabular}{lcccc}
\hline & \multicolumn{3}{c}{ Conglomerado } & \multirow{2}{*}{ Media } \\
\cline { 2 - 4 } & $\mathbf{1}$ & $\mathbf{2}$ & $\mathbf{3}$ & \\
\hline Disfrute & 4.26 & 3.32 & 4.02 & 3.93 \\
Apariencia & 3.97 & 3.24 & 2.45 & 3.31 \\
Social & 4.15 & 2.82 & 3.89 & 3.70 \\
Fitness/Salud & 4.34 & 3.49 & 3.47 & 3.84 \\
Competencia & 4.04 & 2.91 & 3.32 & 3.51 \\
\hline
\end{tabular}

Fuente: elaboración propia.

motivación para la práctica físico-deportiva, obteniendo las puntuaciones de motivación más altas para todos y cada uno de los factores incluidos en la escala. Se trató por tanto del grupo más motivado, tanto intrínseca como extrínsecamente, hacia la práctica físico-deportiva. Dentro de las valoraciones en los diferentes factores, fue la dimensión Fitness/ Salud la que obtuvo una puntuación más alta y la dimensión Apariencia la más baja (Tabla 3; Figura 1).

El clúster 2, constituido por el menor número de casos, $27.2 \%$, se caracterizó porque todos los factores se situaron por debajo de la media. De los tres, fue el conglomerado que obtuvo las puntuaciones más bajas en todos los factores de la escala, salvo en Apariencia y Fitness/Salud. Asimismo, se trató del conglomerado menos motivado intrínsicamente para practicar AF no competitiva. Dentro de este grupo, fueron los factores Fitness/Salud, Disfrute y
Apariencia los que obtuvieron valores más altos, mientras que la dimensión Social y de Competencia obtuvieron los valores más bajos (Tabla 3; Figura1).

Finalmente, el conglomerado 3, conformado por el $30.1 \%$ de la muestra, se distinguió porque los valores medios de los factores Disfrute y Social se encontraron sobre la media del total y el resto de dimensiones estuvieron por debajo de esta. Destacar que la valoración más baja en los factores Apariencia y Fitness/ Salud fue la obtenida en este grupo, aunque de forma más notoria para Apariencia, como se aprecia en la Tabla 3 y en la Figura 1. Estos dos factores, junto con la dimensión Social, están relacionados con la motivación extrínseca hacia la práctica físico-deportiva.

En relación al estudio diferencial de los distintos clusters, los análisis de $\chi^{2}$ realizados para determinar la composición de los clusters en función de las variables sociodemográficas (tipo de organización de

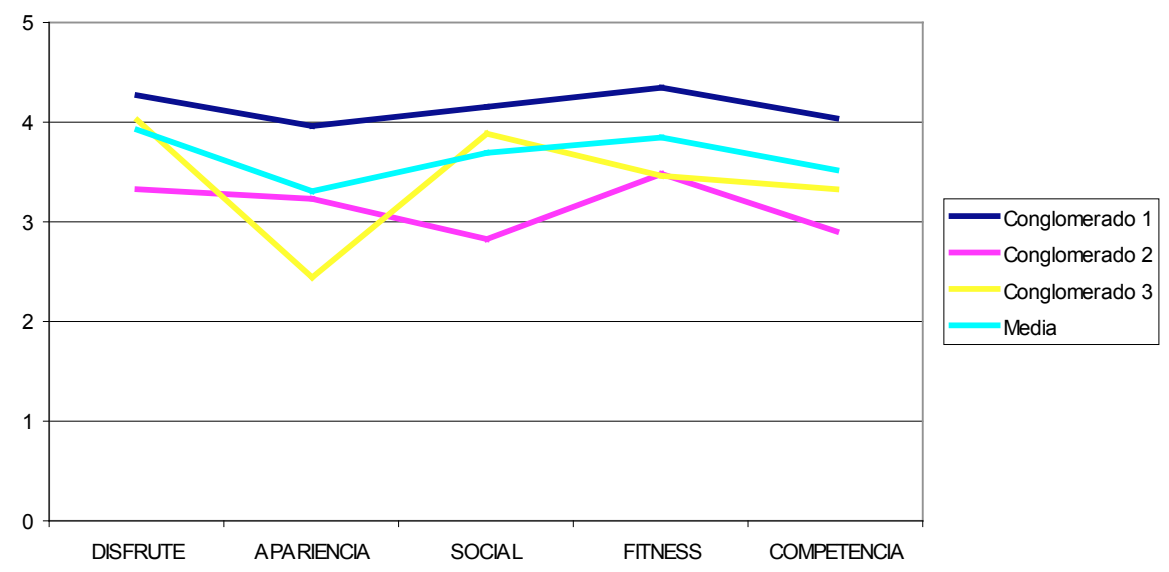

Figura 1. Comparativa de los valores medios obtenidos para cada factor entre la muestra total y la de cada conglomerado. Fuente: elaboración propia. 
pertenencia, sexo, grupo de edad y estudios), mostraron diferencias estadísticamente significativas $(p<0.001)$ en todas ellas (Tabla 4).

En el grupo 1, el más numeroso, se halló el mayor porcentaje de usuarios públicos, así como el de privados, si bien las diferencias no fueron tan acusadas como en los usuarios de organizaciones públicas con respecto a otros conglomerados. El grupo presenta los mayores porcentajes intrasexos, mayor proporción de varones y de mujeres. Los grupos de menor edad (menores de 15 años y sujetos de 15 a 24 años) estuvieron más presentes en este conglomerado que en los dos restantes y, por tanto, su proporción de sujetos con nivel educativo de estudios primarios o secundarios resultó ser mayor que la de aquellos con estudios superiores en relación a los otros conglomerados.

Si se atiende a las características de este conglomerado internamente y no, como en el párrafo precedente, en contraste con los otros dos grupos, se observa que el $56.66 \%$ de los componentes del conglomerado 1, el más motivado hacia la práctica, eran usuarios de servicios deportivos públicos y el $43.34 \%$ de privados. Igualmente, el $52.2 \%$ eran varones, por $47.8 \%$ de mujeres. El $31.22 \%$ de sujetos del conglomerado tenían menos de 15 años, el $28.87 \%$ entre 15 y 24 años, por un $21.04 \%$ que estaba situado entre los 25 y los 45 años, mientras que el $18.87 \%$ superaba los 45 años de edad. En cuanto al nivel educativo, el 35.72 \% tenía un nivel de estudios primarios, el $33.7 \%$ de estudios secundarios, mientras que el $30.58 \%$ tenía estudios superiores.

El conglomerado 2, el menos numeroso y caracterizado por una menor motivación intrínseca que otros grupos, pese a tener menos usuarios de servicios deportivos privados que los otros dos grupos, en términos relativos obtuvo un leve matiz de usuario privado (el $52.13 \%$ de sus componentes eran usuarios de servicios deportivos privados, por el $47.87 \%$ que lo eran de uno público). Al igual, tuvo una mayor proporción de mujeres (del $58.51 \%$ vs. el $41.49 \%$ de varones que conformaron el grupo). Los grupos de edad característicos de este conglomerado fueron los mayores, primando, tanto en relación a otros grupos como internamente dentro del conglomerado 2, los sujetos de 25 a 44 años (33.78 \% de sus componentes) y los mayores de 45 (36.59\% del grupo), mientras que el porcentaje de componentes que tenían menos de 15 años no llegó al $8 \%$. El nivel de estudios fue alto al analizar el grupo internamente, con un $40.03 \%$ de sus componentes con estudios superiores y un $38.49 \%$ con estudios secundarios, por un $21.48 \%$ que solo tiene estudios primarios.

Finalmente, el clúster 3, caracterizado por los niveles más bajos en dos de los tres factores relacionados con la motivación extrínseca, fue el grupo con menor número de usuarios de servicios deportivos públicos y presentó una proporción elevada

TABLA 4

Composición sociodemográfica de los conglomerados y significación de las diferencias entre los mismos

\begin{tabular}{|c|c|c|c|c|c|c|}
\hline & & \multicolumn{3}{|c|}{ Conglomerado } & \multirow{2}{*}{$\chi^{2}$} & \multirow{2}{*}{$p$} \\
\hline & & $1(\%)$ & $2(\%)$ & $3(\%)$ & & \\
\hline \multirow{2}{*}{ Tipo de organización } & Pública & 49.8 & 25.8 & 24.5 & \multirow{2}{*}{34.688} & \multirow{2}{*}{$<0.001$} \\
\hline & Privada & 38.1 & 28.1 & 33.7 & & \\
\hline \multirow{2}{*}{ Sexo } & Varón & 43.9 & 23.9 & 32.2 & \multirow{2}{*}{25.955} & \multirow{2}{*}{$<0.001$} \\
\hline & Mujer & 40.2 & 33.7 & 26.1 & & \\
\hline \multirow{4}{*}{ Edad } & $\leq 15$ & 51.8 & 8.8 & 39.4 & \multirow{4}{*}{157.548} & \multirow{4}{*}{$<0.001$} \\
\hline & $16-24$ & 47.9 & 23.9 & 28.2 & & \\
\hline & $25-45$ & 34.9 & 37.3 & 27.8 & & \\
\hline & $>45$ & 31.3 & 40.4 & 28.4 & & \\
\hline \multirow{3}{*}{ Estudios } & Primarios & 45.9 & 16.8 & 37.4 & \multirow{3}{*}{47.967} & \multirow{3}{*}{$<0.001$} \\
\hline & Secundarios & 43.3 & 30.1 & 26.6 & & \\
\hline & Superiores & 39.3 & 31.3 & 29.4 & & \\
\hline
\end{tabular}

Fuente: elaboración propia. 
de usuarios privados (el $57.9 \%$ de sus componentes eran usuarios de algún servicio deportivo privado, por un $42.1 \%$ restante de uno público), superior a la de los otros dos grupos. En este grupo aumentó el porcentaje de varones y disminuyó el de mujeres con respecto al conglomerado anterior (el $57.8 \%$ de sus componentes fueron varones vs. el $42.2 \%$ de mujeres). La edad de este grupo fue menor que en el grupo 2, con el $31.83 \%$ de sus componentes menores de 15 años, el $22.78 \%$ entre 15 y 24 años, por un $22.45 \%$ de 25 a 45 años y el $22.94 \%$ restante que superaba los 45 años. Mientras el nivel de estudios de sus componentes (el $40.04 \%$ con estudios primarios, el $28.48 \%$ con estudios secundarios y el $31.48 \%$ que había terminado estudios superiores) resultó ser similar al del primer grupo.

\section{Discusión}

Los resultados acerca de los motivos de práctica para el total de la muestra mostraron, en consonancia con la literatura, como las razones de disfrute primaron a la hora de motivar hacia la práctica físico-deportiva (Castillo et al., 2000; García, 2006; Granero-Gallegos et al., 2011; Hellín et al., 2004; Otero, 2009), compartiendo protagonismo con los motivos de salud (Castillo et al., 2000; Fraile \& De Diego, 2006; Moreno et al., 2007; Nuviala et al., 2006; Ruiz et al., 2007). Los motivos de tipo afectivo y social también fueron considerados (García, 2006) y, aunque en menor medida, también los de competencia y criterios estéticos (Granero-Gallegos et al., 2011; Kilpatrick et al., 2005; Olmand et al., 2004). Diferentes investigaciones como la realizada por Castillo et al. (2000), López y Márquez (2001), Sit et al. (2008) y Gómez-López, Ruiz, García, Granero y Piéron (2009) han puesto de manifiesto que el placer y la salud son dos de los principales motivos para practicar actividad física. Estas razones se relacionan con la búsqueda de bienestar y calidad de vida, y favorecen la adherencia al ejercicio físico.

Las dimensiones Apariencia y Social, que han obtenido las puntuaciones medias más bajas, son las que contribuyeron en mayor medida a establecer perfiles motivacionales de práctica de AF. El análisis de conglomerados halló la existencia de tres grupos bien diferenciados, entre los cuales se demostró la existencia de diferencias significativas $(p<0.001)$ en cuanto a las variables sociodemográficas tipo de organización, sexo, edad y nivel de estudios de sus componentes se refiere. Estos resultados no son novedosos, ya que estas variables han sido señaladas por la literatura como factores determinantes en la AF y el deporte (Breuer et al., 2010), existiendo precedentes de significatividad estadística en función de variables de esta naturaleza para los motivos de práctica (Moreno et al., 2007; Ruiz et al., 2007; Sicilia et al., 2009).

El primer perfil motivacional diferenciado (conglomerado 1) se caracterizó por obtener las mayores puntuaciones tanto para las dimensiones de motivación intrínseca como extrínseca, situándose los valores en todas ellas por encima de la media del total de los sujetos objetos de estudio, a pesar de ser inferiores a los que hallan Moreno et al. (2007) en personas adultas, practicantes de AF no competitivas del área metropolitana de una gran ciudad española. Se trata de un grupo altamente motivado por aspectos vinculados a la dimensión Fitness/ Salud, mientras que la dimensión Apariencia es la que obtuvo una valoración más baja.

$\mathrm{Al}$ encuadrarse en este primer grupo la mayor parte de los sujetos, era de esperar que el mismo aglutinase los porcentajes más elevados en las diversas opciones de las variables sociodemográficas estudiadas, como así fue para el tipo de organización (grupo que integró a la mayoría de usuarios tanto públicos como privados), el sexo (mayor cantidad de varones y de mujeres que en otros grupos) y nivel de estudios (acaparó la mayoría de sujetos con estudios primarios, secundarios y superiores), no así para la edad (solo ocurriendo entre los más jóvenes). Sin embargo, el grupo tiene un claro matiz de usuario público y joven, primando aunque en menor medida la cantidad de varones con respecto a la de mujeres y la de usuarios con estudios primarios y secundarios con respecto a los de estudios superiores, pudiéndose atribuir esto último a la juventud de sus componentes. Resultados que han cuestionado a los obtenidos por Moreno et al. (2007) en cuanto a que el factor Fitness/Salud es valorado en mayor medida con el paso de los años, no atribuyendo 
tampoco a los jóvenes las mayores puntuaciones en la dimensión Disfrute. La literatura respalda los resultados de Moreno et al. (2007) en cuanto a la mayor valoración de la salud en las edades más avanzadas (García, 2006; Hellín et al., 2004; Moreno et al., 2007; Otero, 2009; Ruiz et al., 2007; Sit et al., 2008), no así en que los motivos de disfrute sean valorados en otra franja de edad por encima de las puntuaciones de los jóvenes (Castillo et al., 2000; García, 2006; Hellín et al., 2004; Otero, 2009; Ruiz et al., 2007; Sit et al., 2008).

El segundo conglomerado se caracterizó por obtener valores inferiores a la media para la totalidad de las dimensiones, y las más bajas de los tres grupos en los factores Disfrute, Competencia y Social, precisamente los factores que Frederick-Recascino y Shuster-Schmidt (2003) y Ryan et al. (1997) vinculan con una mayor adherencia a la práctica físico-deportiva. Sus componentes estuvieron motivados especialmente por razones vinculadas a las dimensiones Fitness/Salud, Disfrute y Apariencia. Dichos componentes fueron, en su mayoría, usuarios de organizaciones privadas, mujeres y de mayor edad a la del resto de perfiles motivacionales, a lo que puede atribuirse la primacía en este grupo de sujetos con estudios superiores y secundarios a los que poseen primarios. Estos resultados respaldan la importancia de los motivos de salud en sujetos de mayor edad (García, 2006; Hellín et al., 2004; Moreno et al., 2007; Otero, 2009; Ruiz et al., 2007; Sit et al., 2008), así como la relevancia que aún se encuentra entre las edades más avanzadas de las razones lúdicas de práctica físico deportiva (García, 2006; Hellín et al., 2004; Moreno et al., 2007; Otero, 2009; Ruiz et al., 2007). El hecho de encontrar entre las franjas de mayor edad a los sujetos menos motivados parece dar explicación a las mayores tasas de sedentarismo en estas edades (García, 2006, 2010; Otero, 2009).

El tercer y último perfil motivacional encontrado se caracterizó por una motivación basada en las dimensiones Disfrute y Social, únicos factores cuyos valores en este conglomerado estuvieron por encima de la media, resultando ser la dimensión Apariencia la que menos influencia obtuvo en su motivación. Primaron, por tanto, dimensiones relacionadas positivamente con la adherencia deportiva (Frederick-Recascino \& Shuster-Schmidt, 2003; Ryan et al., 1997). El grupo estuvo compuesto principalmente por usuarios privados y, en mayor medida que en el conglomerado precedente, varones jóvenes, en su mayoría menores de 15 años y con estudios primarios. Resultados que coinciden con la literatura al señalar la diversión (Castillo et al., 2000; García, 2006; Hellín et al., 2004; Nuviala et al., 2006; Ruiz et al., 2007; Otero, 2009) y los aspectos sociales (García, 2006) como las principales motivaciones de los jóvenes hacia la $\mathrm{AF}$, así como con la mayor importancia de los aspectos sociales entre los varones, y la menor importancia otorgada a los motivos estéticos en este sexo (García, 2006; Hellín et al., 2004; Moreno et al., 2007; Otero, 2009).

Desde el punto de vista práctico, una de las formas más probadas y aprobadas para conocer un mercado y adaptarse a las necesidades de sus usuarios es segmentarlo en subconjuntos más homogéneos (Bednarik, Šugman, Urank \& Kovač, 2007). $\mathrm{Al}$ respecto, este estudio aporta a las organizaciones deportivas información acerca de la proporción relativa de usuarios con un perfil motivacional determinado y los factores motivacionales característicos de estos perfiles. Del mismo modo también muestra cuáles perfiles son más propensos a encontrarse en una organización privada y en una pública, y cuáles son las características sociodemográficas de los usuarios con cada tipo de perfil. Información que, de acuerdo con autores como Granero-Gallegos et al. (2011), Funk et al. (2009), Moreno et al. (2007) y Sicilia et al. (2009), son de gran ayuda para los gestores deportivos, a la hora de desarrollar estrategias de mercado y publicidad, y mejorar la calidad y satisfacción con el servicio de sus usuarios.

En cuanto a las limitaciones del estudio, destacar que aunque la presente investigación amplía la información proporcionada previamente por investigaciones similares, se debe reconocer la escasa literatura existente sobre la temática tratada, especialmente en revistas internacionales con factor de impacto, máxime si se pretenden comparar resultados obtenidos con el mismo instrumento de medición, lo que ha dificultado la discusión de los 
resultados obtenidos. Serían necesarios futuros estudios que profundicen en este tema y que al mismo tiempo atiendan a otros aspectos condicionantes de la práctica físico-deportiva, como variables socioeconómicas (Breuer et al., 2010) o geográficas (Koo \& Hardin, 2008; Wann et al., 2008; Won \& Kitamura, 2007), así como también que se incluyese una mayor variedad de tipología de organización deportiva.

\section{Conclusiones}

Se parte de la validez y fiabilidad de la escala MPAM-R para la medición de los motivos de práctica en el ámbito de la actividad físico-deportiva no competitiva. Se encontraron tres perfiles de motivación hacia la práctica en la población usuaria de servicios deportivos en Andalucía. Un primer perfil, el más numeroso, caracterizado por haber obtenido los mejores índices de motivación, tanto intrínseca como extrínseca, de los tres grupos, destacando especialmente los motivos de salud, con un matiz de usuario público, varón, joven y con estudios primarios o secundarios. Un segundo perfil, el menos numeroso, con motivación por debajo de la media en todas sus dimensiones, y motivado especialmente por razones de salud, disfrute y apariencia, con un matiz de usuario privado, mujer, de edad media o avanzada y con el nivel de estudios más elevado de los tres grupos. Y un tercer perfil donde priman los motivos de disfrute y sociales, conformado por usuarios en su mayoría de organizaciones privadas, varones jóvenes y con estudios primarios, en su mayor parte.

\section{Referencias}

Aranceta, J., Pérez-Rodrigo, C., Serra-Majem, L., Bellido, D., De la Torre, M. L., Formiguera, X., et al. (2007). Prevention of overweight and obesity: A Spanish approach. Public Health Nutrition, 10(10), 1187-1193.

Beaton, A. A. \& Funk, D. C. (2008). An evaluation of theoretical frameworks for studying physically active leisure. Leisure Sciences, 30(1), 53-70.
Bednarik, J., Šugman, R., Urank, J. \& Kovač, M. (2007). Segmentation of sports consumers in Slovenia. Kinesiology, 39(1), 74-84.

Breuer, C., Hallmann, K., Wicker, P. \& Feiler, S. (2010). Socio-economic patterns of sport demand and ageing. European Reviews of Aging $\mathbb{E}$ Physical Activity, 7(2), 61-70.

Castillo, I., Balaguer, I. \& Duda, J. L. (2000). Las orientaciones de meta y los motivos de práctica deportiva en los jóvenes deportistas valencianos escolarizados. Revista de Psicología del Deporte, 9(1-2), 37-57.

Conroy, D. E., Hyde, A. L., Doerksen, S. E. \& Ribeiro, N. F. (2010). Implicit attitudes and explicit motivation prospectively predict physical activity. Annals of Behavioral Medicine, 39(2), 112-118.

Deci, E. L. \& Ryan, R. M. (2000). The "what" and "why" of goal pursuits: Human needs and the selfdetermination of behaviour. Psychological Inquiry, 11(4), 227-268.

Fraile, A. \& De Diego, R. (2006). Motivaciones de los escolares europeos para la práctica del deporte escolar. Un estudio realizado en España, Italia, Francia y Portugal. Revista Internacional de Sociología, 64(44), 85-109.

Frederick-Recascino, C. M. \& Schuster-Smith, H. (2003). Competition and intrinsic motivation in physical activity: A comparison of two groups. Journal of Sport Behavior, 26(3), 240-254.

Funk, D. C., Filo, K., Beaton, A. A. \& Pritchard, M. (2009). Measuring the motives of sport event attendance: Bridging the academic-practitioner divide to understanding behaviour. Sport Marketing Quartely, 18(3), 126-138.

García, M. (2006). Encuesta sobre los hábitos deportivos de los españoles 2005. Madrid: Consejo Superior de Deportes.

García, M. (2010). Encuesta sobre los hábitos deportivos en España 2010. Avance de resultados. Madrid: Consejo Superior de Deportes.

Gómez-López, M., Ruiz, F., García, M. E., Granero, A. \& Piéron, M. (2009). Motivaciones aludidas por los universitarios que practican actividades físicodeportivas. Revista Latinoamericana de Psicología, 41(3), 519-532.

Granero-Gallegos, A., Gómez-López, M., Abraldes, J. A. \& Rodríguez-Suárez, N. (2011). Motivos de prácti- 
ca en el ámbito de la actividad física no competitiva. Espiral. Cuadernos del Profesorado, 4(7), 15-22.

Guthold, R., Ono, T., Strong, K. L., Chatterji, S. \& Morabia, A. (2008). World-wide variability in physical inactivity: A 51-country survey. American Journal of Preventive Medicine, 34(6), 486-494.

Hellín, P., Moreno, J. A. \& Rodríguez, P. L. (2004). Motivos de práctica físico-deportiva en la Región de Murcia. Cuadernos de Psicología del Deporte, 4(1-2), 101-116.

Kilpatrick, M., Hebert, E. \& Bartholomew, J. (2005). College students' motivation for physical activity: Differentiating men's and women's motives for sport participation and exercise. Journal of American College Health, 54(2), 84-94.

Koo, G. Y. \& Hardin, R. (2008). Difference in interrelationship between spectator motives and behavioural intentions based on emotional attachment. Sport Marketing Quarterly, 17(1), 30-43.

Lobstein, T. \& Millstone, E. (2007). Context for the PorGrow study: Europe's obesity crisis. Obesity Reviews, 8(Suppl. 2), 7-16.

López, C. \& Márquez, S. (2001). Motivación en jóvenes practicantes de lucha leonesa. Revista de Psicología del Deporte, 10(1), 9-22.

Moreno, J. A., Cervelló, E. \& Martínez, A. (2007). Validación de la escala de medida de los motivos para la actividad física-revisada en españoles: diferencias por motivos de participación. Anales de Psicología, 23(1), 167-176.

Moreno, J. A., González-Cutre, C. \& Cervelló, E. (2008). Motivación y salud en la práctica físicodeportiva: diferencias según el consumo de alcohol y tabaco. International Journal of Clinical and Health Psychology, 8(2), 483-494.

Moreno, J. A., Martínez-Galindo, C., González-Cutre, D. \& Marcos, P. (2009). Perfiles motivacionales de practicantes en el medio acuático frente al medio terrestre. Revista Internacional de Medicina y Ciencias de la Actividad Física y el Deporte, 9(34), 201-216.

Nuviala, A., Ruiz, F., García, M. E. \& Díaz, A. (2006). Motivos de práctica y tasa de participación en actividades físico-deportivas organizadas entre adolescentes que viven en dos entornos rurales. Cuadernos de Psicología del Deporte, 6(1), 69-80.
Oldman, A., Daley, A. \& Rickard, N. (2004). Motives for sport and exercise in younger and older half marathon runners. Journal of Sports Sciences, 22(3), 310-311.

Organisation for Economic Co-operation and Development. (2010). Health at a glance: Europe 2010. París: OECD Publishing.

Otero, J. M. (2009). Hábitos y actitudes de la población andaluza ante el deporte 2007. Sevilla: Empresa Pública de Deporte Andaluz.

Rhodes, R. E. \& de Bruijn, G. J. (2010). Automatic and motivational correlates of physical activity: Does intensity moderate the relationship? Behavioral Medicine, 26(2), 44-52.

Rhodes, R. E., Fiala, B. \& Conner, M. (2009). A review and meta-analysis of affective judgments and physical activity in adult populations. Annals of Behavioral Medicine, 38(3), 180-204.

Ruiz, F., García, M. E. \& Díaz, A. (2007). Análisis de las motivaciones de práctica de actividad física y de abandono deportivo en la ciudad de La Habana (Cuba). Anales de Psicología, 23(1), 152-166.

Ryan, R. M., Frederick, C. M., Lepes, D., Rubio, N. \& Sheldon, K. M. (1997). Intrinsic motivation and exercise adherence. International Journal of Sport Psychology, 28, 335-354.

Sicilia, A., Águila, C., Muyor, J. M., Orta, A. \& Moreno, J. A. (2009). Perfiles motivacionales de los usuarios en centros deportivos municipales. Anales de Psicología, 25(1), 160-168.

Sit, C. H. P., Kerr, J. H. \& Wong, I. T. F. (2008). Motives for and barriers to physical activity participation in middle-aged Chinese women. Psychology of Sport and Exercise, 9(3), 266-283.

Wann, D. L., Grieve, F. G., Zapalac, R. K. \& Pease, D. G. (2008). Sport Marketing Quarterly, 17(1), 6-19.

Won, J. \& Kitamura, K. (2007). Comparative analysis of sport consumer motivations between South Korea and Japan. Sport Marketing Quarterly, 16(2), 93-105.

Yan, J. H. \& McCullagh, P. J. (2004). Cultural influence on youth's motivation of participation in physical activity. Journal of Sport Behavior, 27(4), 378-390. 
